\title{
Systematic analysis of porosities in metal-organic frameworks
}

\author{
Kai Trepte ${ }^{1, *}$ and Sebastian Schwalbe ${ }^{2}$ \\ ${ }^{1}$ Department of Physics, Central Michigan University, Mount Pleasant, MI 48859, USA \\ ${ }^{2}$ Institute of Theoretical Physics, TU Bergakademie Freiberg, Leipziger Str. 23, D-09596 Freiberg, Germany
}

(Dated: October 28, 2019)

\begin{abstract}
Porosities of several metal-organic frameworks (MOFs) are computationally analyzed and discussed regarding their sensitivity to numerical parameters. Further, we investigate and discuss the influence of the probe radius on the calculated porosities. A clear differentiation between void and accessible porosity is carried out. This is essential as it impacts the comparison to experimental and theoretical results. We present two different approaches to calculate porosities, and discuss their advantages and drawbacks. All results are compared to well established methods. It will be highlighted that the calculated properties are numerically sensitive to the choice of the used approach and numerical parameters.
\end{abstract}

\section{INTRODUCTION}

Metal-organic frameworks (MOFs) are a material class aiming for different possible applications ${ }^{1}$, such as gas absorbers $^{2,3}$, catalysts $^{4-6}$, optical sensors ${ }^{7,8}$, and postsynthetic modification (PSM) of MOFs for modulating reaction outcomes and biomedical applications ${ }^{9}$. The class of amorphous MOFs (aMOFs) has possible applications as liquids or melt quenched glasses ${ }^{10}$. Recently, it has been shown that the pore sizes in MOFs can be varied by enforcing an external pressure on a given $\mathrm{MOF}^{11}$. Many applications of MOFs are based on the porous nature of these materials, as MOFs typically exhibit several pores. These pores usually have different sizes. With that, an accurate determination of the porosity and the pore sizes is important ${ }^{12}$.

In general, the porosity $\Phi$ is defined as the empty volume $V_{\text {empty }}$ within a given total volume $V_{\text {total }}$ (e.g. the unit cell of a MOF)

$$
\Phi=\frac{V_{\text {empty }}}{V_{\text {total }}} .
$$

While the total volume for crystal structures is always well defined, the empty volume misses this general definition. One major aim of this work is to define and clearly separate two different empty volumes, namely the void volume $V_{\text {void }}$ and the accessible volume $V_{\text {acc }}$. The void volume is the space that is not occupied by any atom in the unit cell. This volume can easily be analyzed given the sizes of the atoms, e.g. their respective van der Waals (vdW) radii ${ }^{13}$. With this volume, the void porosity $\Phi_{\text {void }}$ can be obtained, which serves as a first descriptor of a porous material. However, it has to be considered that a void porosity does not necessarily reflect the volume/porosity which can be assumed by adsorbed species. Such a porosity strictly depends on the size of that species. With that, another volume occurs, i.e. the accessible volume. Accordingly, the accessible

\footnotetext{
*trept1k@cmich.edu
}

porosity $\Phi_{\text {acc }}$ can be defined. This porosity, in contrast to $\Phi_{\text {void }}$, depends on a probe radius $r_{\text {probe }}$ which varies for different species. One has to be careful when analyzing the porosity in a material, as the porosity of interest is usually $\Phi_{\text {acc. }}$. When reporting this quantity, one needs to provide the respective probe radius, such as the vdW radius of $\mathrm{H}\left(r_{\text {probe }}=1.20 \AA\right)$ or Xe $\left(r_{\text {probe }}=2.16 \AA\right)$.

Within this work it will be shown that choosing different probe radii significantly impacts the evaluated porosity. A systematic analysis of the probe radius dependence allows to evaluate the porosity for any adsorbed species, i.e. any atom or molecule with an effective probe radius. Furthermore, additional details about the underlying MOF (i.e. pore sizes) are automatically obtained when using such a systematic study.

This manuscript is structured as follows: in the next section, the theoretical background as well as detailed aspects of the implementation for the different approaches - an overlapping spheres approach (OSA) and a grid point approach (GPA) - are presented. Afterwards, the grid size dependence for the GPA is analyzed for several MOFs. The importance of the probe radius dependence regarding the accessible porosity is discussed. Additional discussions about the computational effort and a comparison to reference values are presented. A conclusion is given in the end.

\section{THEORETICAL BACKGROUND}

We developed the FORTRAN code porE to analyze the porosities and related properties numerically. Only the unit cell parameters and the coordinates of the atoms are needed as input, similar to alternative implementations ${ }^{14-18}$. Two different approaches are implemented, namely an overlapping spheres approach (OSA) and a grid point approach (GPA). While the OSA is very fast and gives a good approximation for the (void) porosity, the GPA is able to distinguish between void and accessible properties and can be tuned to any desired accuracy. Both approaches are summarized below.

For this study, the MOFs UiO- $66^{19-21}$, UiO- $67^{20-22}$, 
DUT-8 $(\mathrm{Ni})_{\text {open }}{ }^{23-25}$, DUT-8 $(\mathrm{Ni})_{\text {closed }^{23}}{ }^{23}$ IRMOF-10 $0^{26}$, MOF- $5^{27}$, HKUST- $1^{28-30}$ and MOF $210^{31}$ are investigated. For convenience, the two structures (open, closed) of DUT- $8(\mathrm{Ni})$ are abbreviated with DUT- $8(\mathrm{Ni})_{\mathrm{o}}$ and DUT- $8(\mathrm{Ni})_{c}$. Further, MOF-210 is only analyzed with the OSA, as its structure is currently to large to be properly analyzed with the GPA.

For illustration, pore centers determined using porE are plotted as spheres within the periodic structures of the investigated MOFs (see Fig. 1).

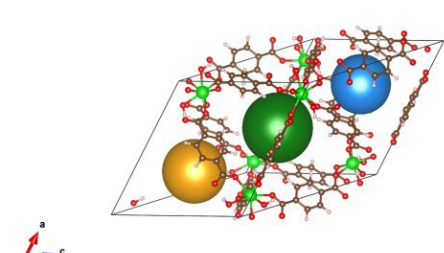

$\mathrm{C}_{\mathrm{b}}^{\mathrm{c}}$
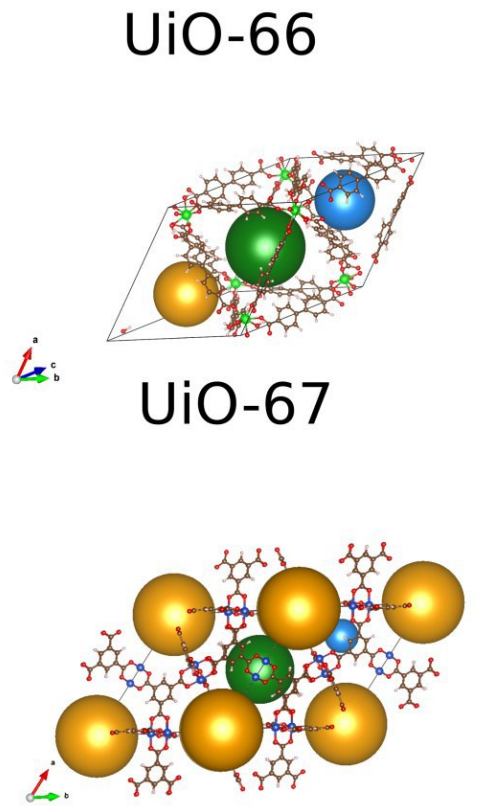

HKUST-1

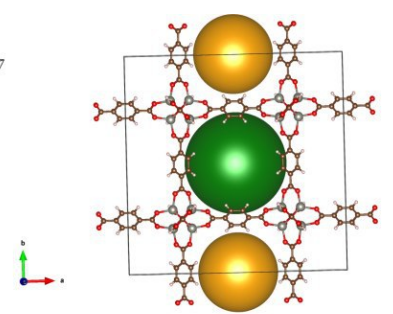

MOF-5
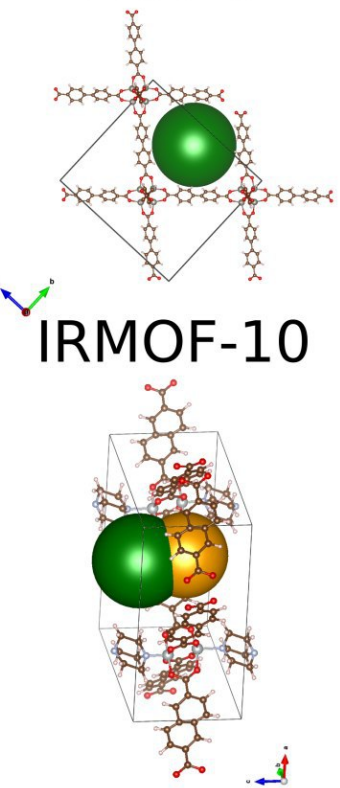

DUT-8 $(\mathrm{Ni})$ 。
FIG. 1. Pores visualized as spheres for the test set of MOFs. HKUST-1, UiO-66, and UiO-67 have three distinct pores. DUT- $8(\mathrm{Ni})_{0}$ and MOF-5 have two characteristic pores, while IRMOF-10 only has one pore, which is repeated within the periodic structure. The individual pictures are generated with VESTA using the calculated pore centers as additional species with an effective radius equal to the determined pore size. The entire picture was generated using the inkscape program.

\section{A. Overlapping sphere approach (OSA)}

To evaluate the porosity within MOFs (or any porous material), a hard sphere model can be used. In such a model, the overlap of atomic spheres is evaluated and consequently subtracted from the total volume of all spheres/atoms. The volume which is left, $V_{\text {void }}$, can be compared to the total volume of a unit cell, providing the (void) porosity $\Phi_{\text {void }}$ as

$$
\Phi_{\text {void }}=\frac{V_{\text {void }}}{V_{\text {total }}}
$$

with the void, total, occupied, atomic and overlap volumes given by

$$
\begin{aligned}
V_{\text {void }} & =V_{\text {total }}-V_{\text {occ }} \\
V_{\text {total }} & =\mathbf{a} \cdot(\mathbf{b} \times \mathbf{c}) \\
V_{\text {occ }} & =V_{\text {atoms }}-V_{\text {overlap }} \\
V_{\text {atoms }} & =\sum_{i} V_{i, \mathrm{vdW}} \\
V_{\text {overlap }} & =\sum_{i, j>i} V_{i j, \text { overlap }}
\end{aligned}
$$

Here, $\mathbf{a}, \mathbf{b}$ and $\mathbf{c}$ are the unit cell vectors and $V_{i, \mathrm{vdW}}$ is the volume of a sphere with a radius equal to the vdW radius of atom $i$. The sum of the volumes of all atoms $V_{\text {atoms }}$ minus the overlap $V_{\text {overlap }}$ between pairs of atoms defines the occupied volume $V_{\text {occ }}$. The overlap volume can be calculated analytically following the derivation in the supplemental material. This is done if the sum of the covalent radii is smaller than the distance between atoms $i$ and $j$, thus if $r_{i}^{\text {covalent }}+r_{j}^{\text {covalent }} \leq d_{i j}$. This simple approach gives reasonable results (see Tab. I, a comparison to literature values is given in Tab. III) at essentially no computational cost.

TABLE I. All volumes (in $\AA^{3}$ ) for the determination of the porosity (in \%) of all considered MOFs based on the simple hard sphere approach (OSA).

\begin{tabular}{l|rrrrrr} 
MOF & $V_{\text {total }}$ & $V_{\text {atoms }}$ & $V_{\text {overlap }}$ & $V_{\text {occ }}$ & $V_{\text {void }}$ & $\Phi_{\text {void }}$ \\
\hline DUT-8(Ni) & 3190 & 1953 & 709 & 1244 & 1946 & 61 \\
DUT-8(Ni)c & 648 & 976 & 324 & 652 & -4 & -1 \\
UiO-66 & 2308 & 1992 & 823 & 1169 & 1139 & 49 \\
UiO-67 & 4972 & 2906 & 1308 & 1599 & 3374 & 68 \\
IRMOF-10 & 10099 & 2549 & 1297 & 1252 & 8847 & 88 \\
MOF-5 & 17305 & 6536 & 2592 & 3944 & 13362 & 77 \\
HKUST-1 & 4546 & 2499 & 1062 & 1437 & 3109 & 68 \\
MOF210 & 144400 & 29385 & 14937 & 14449 & 129952 & 90
\end{tabular}

As higher-order terms are neglected, only the twocenter overlap is calculated. However, the OSA recovers the total overlap (three-order and higher terms) almost entirely for the benzene molecule. The literature value ${ }^{32}$ of the occupied volume in benzene, treating only the C-C overlap, is $V_{\text {occ }}^{\text {ref }}=114.8 \AA^{3}$. The proposed OSA delivers a value of $V_{\text {occ }}^{\mathrm{OSA}}=115.2 \AA^{3}$. For comparison, the sum of the vdW spheres of all atoms is $166.9 \AA^{3}$. The molecular geometry is taken from the $\mathrm{CCCBDB}^{33}$ with $d_{\mathrm{C}-\mathrm{C}}=1.397 \AA$, in analogy to Gibson and Scheraga ${ }^{[32]}$. Given this result, it can be assumed that the results for the overlap should be accurate, even without the higherorder terms. 
The main advantage of the OSA is the access of reasonable results with essentially no numerical effort. For example, the calculation for MOF210 (1854 atoms per unit cell) takes about $1 \mathrm{~s}$ (see supplemental material). The main disadvantage is that technically only the void porosity is calculated and there is no information about accessible terms. The approach presented in the next section overcomes this shortcoming.

\section{B. Grid point approach (GPA)}

An alternative approach is based on a numerical grid inside the unit cell. This procedures requires the explicit treatment of each grid point. Any grid point is either close to an atom (inside its vdW sphere) and can be considered occupied. If no such occupation is found, the grid point is considered unoccupied. In analogy to equation (2), the void porosity can be evaluated by the number of unoccupied points divided by the total number of grid points

$$
\Phi_{\text {void }}=\frac{N_{\text {unoccupied }}}{N_{\text {total }}}
$$

A suitable amount of grid points will provide accurate results. Using this ansatz, one obtains an insight into the void volume and thus the void porosity. This is not equivalent to the accessible volume and porosity, which is often given in the literature. Thus, one needs to be careful when comparing e.g. the values for DUT- $8(\mathrm{Ni})_{\text {closed }}$ as explained in the supplemental material.

The accessible volume can be obtained by modifications to the presented ansatz. Grid points need to be evaluated such that around each grid point, a sphere with a probe radius $r_{\text {probe }}$ is assumed. If this sphere has no contact with the vdW surface of the MOF, all points inside this sphere are considered to be unoccupied as well as accessible. With that, points can be occupied, unoccupied and not accessible or unoccupied and accessible (see Fig. 2). This ansatz gives rise to another quantity, the accessible porosity $\Phi_{\text {acc }}$, which depends on the probe radius $\Phi_{\text {acc }}=\Phi_{\text {acc }}\left(r_{\text {probe }}\right)$. The relation $\Phi_{\text {acc }}\left(r_{\text {probe }}\right) \leq \Phi_{\text {void }}$ is clearly fulfilled. The basic outline of the procedure is given in Fig. 3.

The used grid is defined either as a total number of grid points for each cell vectors or as an approximate grid density per $\AA$ for all cell vectors. In either case, the grid points are placed along the cell vectors. With that, we have a unique grid for any system. This grid can either be uniform (grid density along all cell vectors is the same) or non-uniform. In this work, we only investigate uniform grids. It should be noted that the accessible pore windows, i.e. the maximum size to access a pore at all, has not been investigated. While other codes like Zeo $++{ }^{18,34-37}$ provide such an analysis which is of importance regarding a better comparison with e.g. experimental values, the main goals of this manuscript

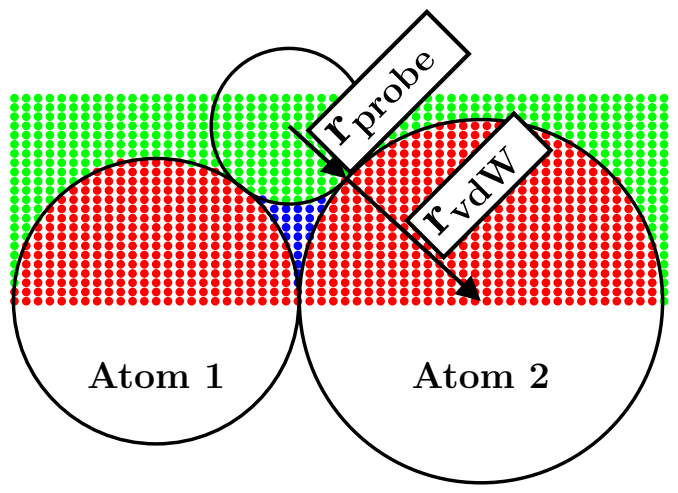

FIG. 2. Visual explanation of different grid points in the GPA. If a grid point is inside the vdW sphere of an atom, it is occupied (red). Otherwise, it is unoccupied. Then, two different cases can occur. If the point is in a region which can be accessed as described by a given probe radius $r_{\text {probe }}$ (green), it is unoccupied and accessible. Otherwise, it is unoccupied and not accessible (blue). The difference in the blue and the green points defines the difference between the void and the accessible porosity.

are unaffected by not analyzing this property. An analysis will be provided in future versions of the code. The effect of studying the pore windows on the porosities was investigated using Zeo++, see supplemental material.

From a numerical point of view, the variable $N_{\text {check_acc }}$ is introduced (see Fig. 3). With this variable, there is no longer the need to loop over all accessible points to determine which unoccupied points are also accessible. Only selected points have to be evaluated (see Fig. 4). This reduces the computational time while not changing the results. The variable $N_{\text {check_acc }}$ is defined as a subset of points chosen from all immediately accessible points, i.e. all points with a distance larger than $r_{\mathrm{vdW}}+r_{\text {probe }}$ for all atoms. This subset contains points which have a distance within $r_{\mathrm{vdW}}+\delta$, with $\delta=r_{\text {probe }} \cdot(1.0+h)$, $h=1.0 / \bar{n}$ and $\bar{n}$ is the average grid point density per $\AA$. Accordingly, $\delta$ becomes smaller for larger grids. All points $k$ within this subset are within a distance of $r_{\mathrm{vdW}}+r_{\text {probe }} \leq d_{k} \leq r_{\mathrm{vdW}}+\delta$. Visually, this subset forms a layer of thickness $\delta-r_{\text {probe }}=r_{\text {probe }} \cdot h=r_{\text {probe }} / \bar{n}$ over the vdW surface of the MOF (indicated in gray in Fig. 4).

The obtained subset $\left(N_{\text {check_acc }}\right)$ is used to identify whether unoccupied points are also accessible. If the distance of any unoccupied point to any point in $N_{\text {check_acc }}$ is smaller than $r_{\text {probe }}$, the unoccupied point is accessible (green point in Fig. 4). Otherwise, the point is not accessible (blue point in Fig. 4). Further modifications to this approach can be introduced, where $N_{\text {check_acc }}$ is only obtained per atom. If an unoccupied point is close to an atom $i$, only the points $N_{\text {check_acc, } i}$ need to be evaluated. This is the basis of GPA sub-grid $_{\text {(see section Computa- }}$ tional Time and the supplemental material), which gives an additional speed-up. 


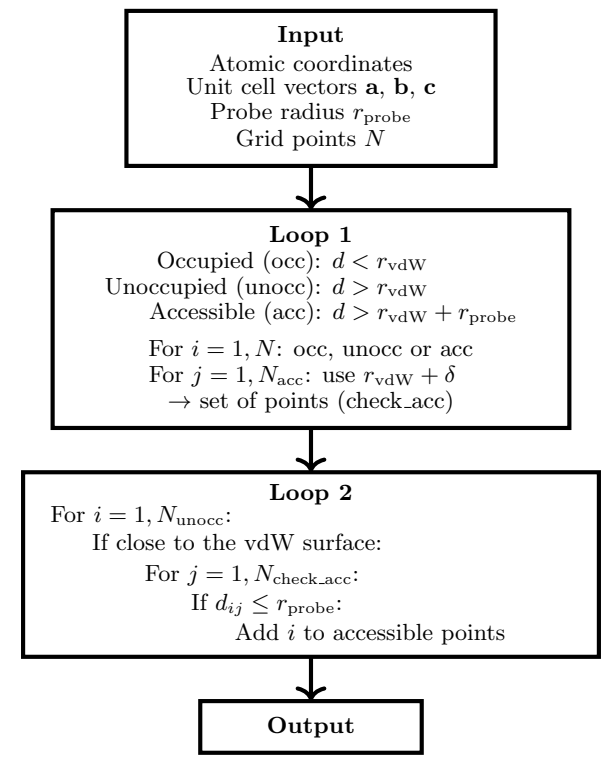

FIG. 3. Outline of the grid point approach (GPA) to evaluate void and accessible porosities (and related properties). In loop 1, everything regarding the void porosity is collected (occupied, unoccupied and all immediately accessible points). During loop 2, it is determined which unoccupied points are also accessible. This ensures that all remaining accessible points are collected for the accessible porosity. Here, $\delta=$ $r_{\text {probe }} \cdot(1.0+h)$, with $h=1.0 / \bar{n}$ and $\bar{n}=\left(n_{x}+n_{y}+n_{z}\right) / 3$ being the average grid point density per $\AA$ in all directions. Thus, the denser the grid, the smaller $\delta$.

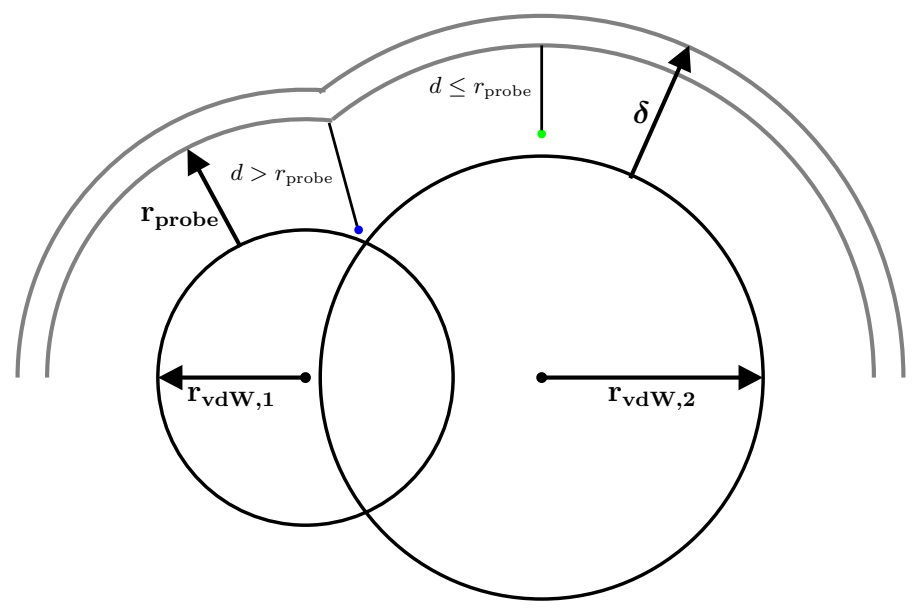

FIG. 4. Visualization of $N_{\text {check_acc. Circles for Atom } 1 \text { and }}$ Atom 2 indicate their respective vdW radii $r_{\mathrm{vdW}}$. The subset of points $N_{\text {check_acc }}$ is chosen from all immediately accessible points, i.e. all points with a distance larger than $r_{\mathrm{vdW}}+r_{\text {probe }}$ for all atoms. This subset (indicated in gray) contains points which have a distance within $r_{\mathrm{vdW}}+\delta$, where $\delta=r_{\text {probe }}$. $(1.0+h), h=1.0 / \bar{n}$ and $\bar{n}$ is the average grid point density per $\AA$. Accordingly, $\delta$ becomes smaller for larger grids. If the distance of an unoccupied point to any $N_{\text {check_acc }}$ is smaller than $r_{\text {probe }}$, this point is also accessible (green). Otherwise, it is not accessible (blue, color code adopted from Fig. 2).

\section{GRID SIZE DEPENDENCE}

As the GPA depends on the (uniform) distribution of grid points, it is important to determine how dense the grid needs to be to provide numerically reliable results. For all structures (except MOF210), the grid was successively increased and the porosities (void and accessible) were calculated. A probe radius of $1.20 \AA$ was used for all MOFs. In addition, a probe radius of $2.16 \AA$ has been employed for DUT-8 $(\mathrm{Ni})_{\text {open }}, \mathrm{UiO}-66$ and UiO-67. This was done to see whether the porosities converge differently using different probe radii. The results for all MOFs are summarized in the supplemental material. For UiO-66, the results are given in Fig. 5 .

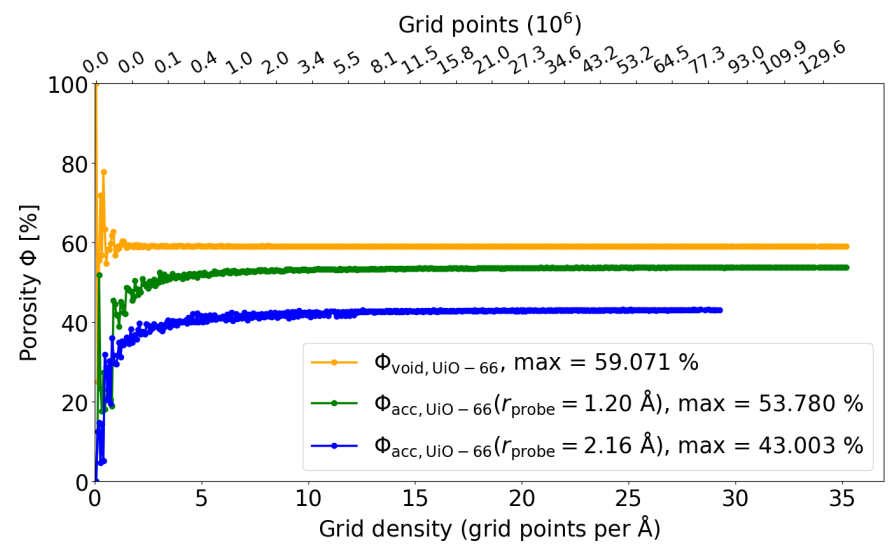

FIG. 5. Grid size dependence for UiO-66. The void porosity as well as the accessible porosity for two different probe radii are shown. The $\mathrm{x}$-axes denote the used grid. Maximum grid: $\approx 35 / \AA \rightarrow 1.42 \cdot 10^{8}$ points for $r_{\text {probe }}=1.20 \AA$ and $\approx 29 / \AA \rightarrow$ $8.17 \cdot 10^{7}$ points for $r_{\text {probe }}=2.16 \AA$.

Clearly, the void porosity converges very fast with an increasing grid, and even a smaller number of grid points provides good results. For the presented example, a grid point density of 5 points $/ \AA$ seems to be sufficient for the void porosity. On the other hand, the accessible porosity converges much slower, and more grid points are needed to reach convergence. Using our example, at least 10 points $/ \AA$ are needed to sufficiently converge the results. This is true for the other MOFs as well.

Using different probe radii influence the convergence as well. Furthermore, the accessible porosity for the different probe radii is very different. This will be discussed in the next section in detail.

As a note here, the results can be systematically improved by using larger grids.

\section{PROBE RADIUS DEPENDENCE}

After establishing that a grid size of ca. 10 points $/ \AA$ is sufficient for an accurate description of the porosities, the next question is how the accessible porosity changes 
for different probe radii. This becomes especially important if the porosity is analyzed with respect to different adsorbed species, having different effective probe radii. Furthermore, it is important to analyze this behavior regarding the comparison with literature values. Usually, the accessible porosity is reported, while the probe radius is typically disregarded. Here we show that the accessible porosity strongly depends on the probe radius, which can be seen for all MOFs in Fig. 6. Individual pictures and values for each MOF are given in the supplemental material.

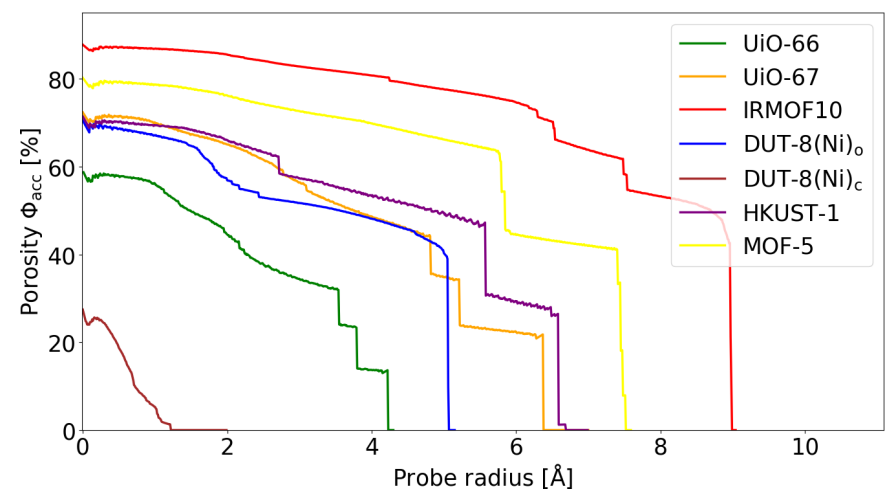

FIG. 6. Probe radius dependence for some MOFs. The accessible porosity is given on the y-axis, while the probe radius is shown on the x-axis. The used grid is approximately 10 points/ $\AA$ for all MOFs besides UiO-66 (12.5 points/ $\AA$ ) and DUT- $8(\mathrm{Ni})_{\text {closed }}(20$ points $/ \AA)$. The drops in the accessible porosity correspond to different pore sizes/radii.

Fig. 6 should make it clear that different species have different accessible porosities. For example, the vdW radius of a Xe atom is $2.16 \AA$, the one of $\mathrm{CH}_{4}$ is $\approx 2.29 \AA$, the one for $\mathrm{SO}_{4}^{2-}$ is $\approx 3.01 \AA$ and the one for $\mathrm{C}_{2} \mathrm{H}_{6}$ is $\approx 5.48 \AA$. These values were determined by using the bond distances and the vdW radii of the atoms. For $\mathrm{C}_{2} \mathrm{H}_{6}$, the distance between the most distant $\mathrm{H}$ atoms was used. It should be noted that all molecules are approximated as spheres having some effective probe radius. The goal here is to make the comparison and the used probe radii more intuitive to understand. This shall not be interpreted as an accurate approximation for e.g. adsorption investigations (clearly, $\mathrm{C}_{2} \mathrm{H}_{6}$ is not spherical and shape effects become important), but only as a way to analyze the probe radius dependence. The accessible porosity for such species will be smaller than for e.g. a $\mathrm{H}\left(r_{\text {probe }}=1.20 \AA\right)$ or a He atom $\left(r_{\text {probe }}=1.40 \AA\right)$. To illustrate this more quantitatively, the accessible porosities for different probe radii are listed in Tab. II. The same analysis was done with Platon ${ }^{14}$ and Zeo $++{ }^{18,34-37}$ (see supplemental material). The general trends for the probe radius dependence for all investigated MOF are consistent between the used codes. As an example, the accessible porosity for UiO-66 behaves as follows: $59.1 \%\left(r_{\text {probe }}=0.00 \AA\right)$ to $53.3 \%\left(r_{\text {probe }}=1.20 \AA\right)$ to $50.8 \%\left(r_{\text {probe }}=1.40 \AA\right)$ to $42.9 \%\left(r_{\text {probe }}=2.16 \AA\right)$ to $39.9 \%\left(r_{\text {probe }}=2.29 \AA\right)$ to $34.2 \%\left(r_{\text {probe }}=3.01 \AA\right)$ to $0.0 \%\left(r_{\text {probe }}=5.48 \AA\right)$. Furthermore, it should be mentioned that the void porosity is recovered for $r_{\text {probe }}=0.00 \AA$. In addition, the accessible porosity has to become zero for $r_{\text {probe }} \rightarrow \infty$.

TABLE II. Accessible porosities (in \%) for some MOFs depending on different probe radii (in $\AA$ ). The used grid contains ca. 10 points $/ \AA$, except for UiO-66 where the grid point density is 12.5 points $/ \AA$.

\begin{tabular}{|c|c|c|c|c|c|c|c|}
\hline$r_{\text {probe }}$ & 0.00 & 1.20 & 1.40 & 2.16 & 2.29 & 3.01 & 5.48 \\
\hline DUT-8(Ni)o & 70.5 & 66.3 & 65.6 & 55.7 & 54.5 & 51.7 & 0.0 \\
UiO-66 & 59.1 & 53.3 & 50.8 & 42.9 & 39.9 & 34.2 & 0.0 \\
UiO-67 & 72.4 & 68.8 & 67.8 & 64.1 & 63.2 & 56.4 & 23.2 \\
IRMOF-10 & 87.8 & 86.6 & 86.5 & 85.0 & 84.6 & 82.8 & 76.4 \\
MOF-5 & 80.2 & 78.3 & 77.9 & 75.4 & 75.0 & 72.6 & 64.5 \\
HKUST-1 & 71.4 & 69.1 & 68.9 & 65.1 & 64.6 & 57.3 & 47.0 \\
\hline
\end{tabular}

Besides its fundamental importance, one can use a screening of different probe radii to analyze the pore sizes of MOFs. First, one can calculate the porosities for different probe radii. Then, the accessible porosity is monitored. Once the probe radius is larger than a specific pore, all grid points within this pore become not accessible. Accordingly, the accessible porosity drops significantly. This gives an intuitive way to characterize the pore sizes (see Fig. 6). More information is given in the supplemental material.

Furthermore, any effective probe radius corresponding to any molecule, e.g. $\mathrm{CH}_{4}$, can be analyzed regarding the porosity. With that, one can define a porosity for any species, and check whether it fits into specific pores of a MOF or not.

A different approach to analyze the pore dimensions/ pore size distribution is discussed in the supplemental material.

\section{COMPUTATIONAL TIME}

Considering the grid point approach, the computational time for any structure which has a certain amount of accessible volume should scale roughly like $N^{2}$, with $N$ being the number of grid points. This is due to loop 2 (see Fig. 3). For structures without any accessible volume, there is nothing to be done in loop 2 and the computational time should scale roughly linearly with $N$. This trend is found, as can be seen for UiO-66 in Fig. 7. Computational times for all MOFs can be found in the supplemental material, where additional discussions regarding the dependence of the computational time with respect to the probe radius are given.

To make the GPA more efficient, a modification was introduced. The idea is to sub-divide the $N_{\text {check_acc }}$ grid points into atom-centered sub-grids. With that, if an unoccupied point is close to atom $i$, only $N_{\text {check_acc, } i}$ grid points are needed in loop 2. More details are given in the supplemental material. Using this alternative approach 


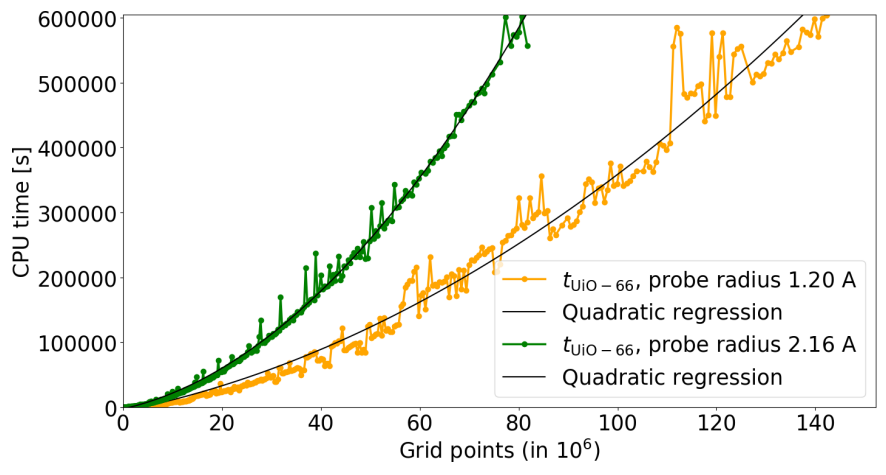

FIG. 7. Computational times for the calculation of the porosities for UiO-66 using two different probe radii and an increasing amount of grid points. A typical grid density of 5 points $/ \AA$ corresponds to $\approx 4.2 \cdot 10^{5}$ grid points, and the respective calculation takes about $68 \mathrm{~s}$.

$\left(\mathrm{GPA}_{\text {sub-grid }}\right)$, the computational time is drastically reduces and a near-linear dependence of the computational time with respect to the grid is achieved. An example for these computational times using $\mathrm{UiO}-66$ is given in Fig. 8.

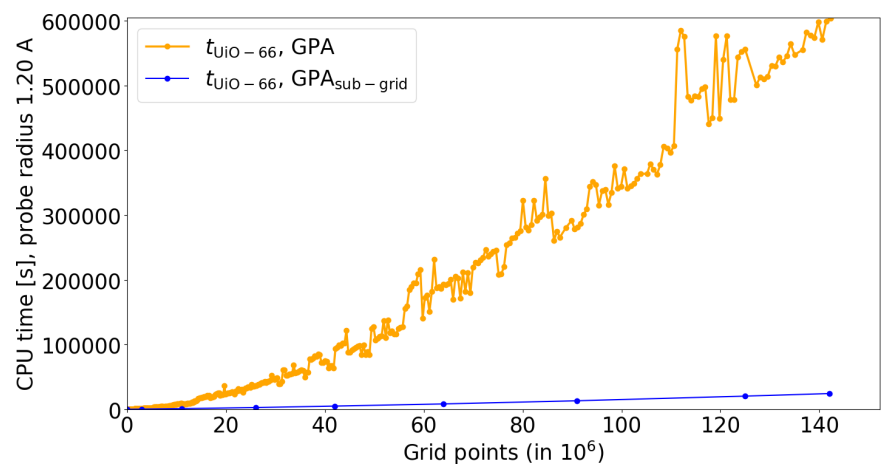

FIG. 8. Computational times for the GPA in UiO-66 using a probe radius of $1.20 \AA$ and an increasing amount of grid points. A comparison between GPA and GPA sub-grid is carried out. With the latter, a near-linear behaviour is achieved.

\section{COMPARISON TO REFERENCES}

To validate the implementation in porE, several reference calculations were performed. For this, we used the codes RASPA2 ${ }^{16,17}$, Poreblazer ${ }^{15}$, Platon ${ }^{14}$ and Zeo $++^{18,34-37}$.

The RASPA2 code provides various features for the calculation of porous materials. The porosity can be accessed using the calculation mode for the determination of the helium void fraction. For that, the porous structure is probed with a helium atom at room temperature. The helium atom itself is described with a TraPPE force field ${ }^{16,17,40}$, while the porous structure can be described with various available force fields. For our reference calculations, we used the CrystalGenerator force
TABLE III. Comparison of calculated porosities between reference codes $\left(\mathrm{RASPA} 2^{16,17}(\pi)\right.$, Poreblazer $^{15}(\rho)$, Platon $^{14}(\gamma)$, Zeo $\left.++{ }^{18,34-37}(\xi)\right)$, literature values and the presented approaches (OSA $(\alpha)$ and GPA $(\omega))$. For the GPA, a differentiation between void and accessible porosities (using $r_{\text {probe }}=1.20 \AA$ and the largest possible grids) is done. All porosities are given in \%. The literature values (REF) are: DUT- $8(\mathrm{Ni})^{23}$, UiO-66 and $\mathrm{UiO}-67^{38}$, IRMOF-10 ${ }^{26}$, MOF5 and MOF210 ${ }^{31}$, HKUST- $1^{12,39}$.

\begin{tabular}{l|rrrr|rrr|r} 
MOF & $\pi$ & $\rho$ & $\gamma$ & $\xi$ & $\alpha$ & $\omega_{\text {void }}$ & $\omega_{\text {acc }}$ & $\Phi_{\text {REF }}$ \\
\hline DUT-8(Ni) & 70 & 68 & 66 & 68 & 61 & 70 & 67 & 67 \\
DUT-8(Ni) & 0 & 0 & 0 & 0 & -1 & 27 & 1 & 0 \\
UiO-66 & 52 & 51 & 53 & 55 & 49 & 59 & 54 & 53 \\
UiO-67 & 72 & 72 & 69 & 70 & 68 & 72 & 69 & 68 \\
IRMOF-10 & 91 & 90 & 86 & 87 & 88 & 88 & 87 & 87 \\
MOF-5 & 81 & 81 & 76 & 79 & 77 & 80 & 79 & 79 \\
HKUST-1 & 73 & 72 & 69 & 69 & 68 & 71 & 69 & 68 \\
MOF210 & 93 & - & -88 & 90 & - & - & 89
\end{tabular}

field $^{16,17}$, 2000 Monte Carlo cycles and the unit cell of the MOFs. The used unit cells contain the following amount of atoms: DUT- $8(\mathrm{Ni})_{\text {open }}$ (132), DUT- $8(\mathrm{Ni})_{\text {closed }}(66)$, UiO-66 (114), UiO-67 (174), HKUST-1 (156), IRMOF10 (166), MOF-5 (424) and MOF210 (1854). All structures are available at https://github.com/kaitrepte/ porE. The Platon code offers several analysis techniques for crystal structures. It allows to analyze the accessible porosity using a grid based approach, similar to the GPA presented here. The Poreblazer code can be used to analyze the surface areas, the pore size distribution and the porosity. In Poreblazer, the porous system is describe using the universal force field $\left(\mathrm{UFF}^{41}\right)$ and the helium atoms are describe using a Lennard-Jones force field description. The (helium) void volume is calculated using a cublet procedure. In Zeo++, the accessible volume is calculated by placing points randomly in the unit cell. Afterwards, each point is analyzed regarding its accessibility with respect to a given probe radius using a Voronoi decomposition scheme ${ }^{18}$.

In general the void porosities using porE with the GPA agree well with the results of the RASPA2 and the Poreblazer code (see Tab. III). The accessible porosities applying porE with the GPA for the set of MOFs is in excellent agreement with the results calculated with the Platon and the Zeo++ codes (see Tab. III). Further, a comparison to literature values is carried out. Here, one essential point is that literature values are accessible porosities, while the corresponding probe radius is usually not provided. Clearly, the void porosities do not accurately reflect the porous nature of the MOFs.

This is especially true for systems with more complex pores. There, the probe radius plays an even more crucial role (compare e.g. UiO-66 and MOF-5). In MOFs with large, open pores the void porosity will already reflect the porous nature of the MOF fairly accurately. If a pore would be entirely spherical, i.e. the pore would have a spherical symmetry, the calculated porosity would be independent of the probe radius (unless $r_{\text {probe }} \geq r_{\text {pore }}$ ). Us- 
ing any probe radius smaller than the pore radius would sample the pore entirely, as there are no areas/volumes which are inaccessible. With that, the porosity is independent of the probe radius and the void porosity is already a good quantity.

However, if the pores are different from the spherical symmetry, e.g. having tetrahedral or octahedral symmetries as in the $\mathrm{UiOs}$, the probe radius dependence is significantly larger. In such a case, a sphere is not a suitable approximation for the pores (see Fig. 1 for UiO-66 and UiO-67). Quantitatively, this can be seen e.g. for UiO-66 (see table III), where the void and the accessible porosities are quite different (59\% and $54 \%$ ). This is true even at a fairly small probe radius of $1.20 \AA$. The dependence becomes clearer the larger the probe radius becomes. Further, this explains the rather small dependence of the accessible porosity for $r_{\text {probe }}=1.20 \AA$ in the MOFs IRMOF-10, MOF-5 and HKUST-1 (see table II and the supplemental material), as for these three MOFs spheres are a fair approximation to describe the respective pores.

In summary, the accessible porosities from the GPA are in excellent agreement with literature values, reassuring a proper implementation. Considering its limitations, even the OSA provides reasonable results.

\section{CONCLUSION}

We present two approaches to analyze the porosity in porous materials, in specific for metal-organic frameworks (MOFs). Various MOFs (DUT-8(Ni) open,
DUT-8(Ni) $)_{\text {closed }}, \mathrm{UiO}-66, \mathrm{UiO}-67$, IRMOF-10, MOF-5, HKUST-1, MOF210) have been studied using these approaches. One approach is based on overlapping spheres (OSA) and their corresponding volumes, from which the void porosity is calculated. This approach is computationally very efficient. The other approach (GPA) uses a grid within the unit cell. With this approach, a clear differentiation between accessible and void porosities can be made. It was shown that it is essential to converge the results with respect to the used grid. Further, the crucial importance of a clear differentiation between accessible and void porosity for the correct description of porosities in MOFs was demonstrated. For the accessible porosity, it was shown that a correct treatment of the probe radius is essential for reliable results. Thus, this dependence must be considered in any case. A comparison to reference calculations and literature values confirms that both approaches work as intended, and that the GPA gives very accurate results. The poreE code developed for all investigations and used for all primary calculations can be found under OpenSource licence (Apache 2.0) at github:

https://github.com/kaitrepte/porE.

\section{CONFLICTS OF INTEREST}

There are no conflicts of interest to declare.

\section{ACKNOWLEDGEMENT}

The authors thank the ZIH in Dresden and the HPCC at MSU for computational time and support.
[1] S. Yuan, L. Feng, K. Wang, J. Pang, M. Bosch, C. Lollar, Y. Sun, J. Qin, X. Yang, P. Zhang, Q. Wang, L. Zou, Y. Zhang, L. Zhang, Y. Fang, J. Li, and H.-C. Zhou, Advanced Materials 30, 1704303 (2018).

[2] J.-R. Li, R. J. Kuppler, and H.-C. Zhou, Chemical Society Reviews 38, 1477 (2009).

[3] H. Li, K. Wang, Y. Sun, C. T. Lollar, J. Li, and H.-C. Zhou, Materials Today 21, 108 (2018).

[4] F. X. L. i Xamena, A. Abad, A. Corma, and H. Garcia, Journal of Catalysis 250, 294 (2007).

[5] L. Jiao, Y. Wang, H.-L. Jiang, and Q. Xu, Advanced Materials 30, 1703663 (2018).

[6] F.-L. Li, Q. Shao, X. Huang, and J.-P. Lang, Angewandte Chemie International Edition 57, 1888 (2018).

[7] J. Lei, R. Qian, P. Ling, L. Cui, and H. Ju, TrAC Trends in Analytical Chemistry 58, 71 (2014), new Nanobiosensing Techniques and Bioanalysis.

[8] Y. Zhang, S. Yuan, G. Day, X. Wang, X. Yang, and H.-C. Zhou, Coordination Chemistry Reviews 354, 28 (2018).

[9] S. M. Cohen, Chemical Science 1, 32 (2010).

[10] T. D. Bennett and A. K. Cheetham, Accounts of Chemical Research 47, 1555 (2014).

[11] S. A. Moggach, T. D. Bennett, and A. K. Cheetham, Angewandte Chemie International Edition 48, 7087
(2009).

[12] D. Ongari, P. G. Boyd, S. Barthel, M. Witman, M. Haranczyk, and B. Smit, Langmuir 33, 14529 (2017).

[13] A. Bondi, The Journal of Physical Chemistry 68, 441 (1964).

[14] A. L. Spek, Acta Crystallographica Section D 65, 148 (2009).

[15] L. Sarkisov and A. Harrison, Molecular Simulation 37, 1248 (2011).

[16] D. Dubbeldam, A. Torres-Knoop, and K. S. Walton, Molecular Simulation 39, 1253 (2013).

[17] D. Dubbeldam, S. Calero, D. E. Ellis, and R. Q. Snurr, Molecular Simulation 42, 81 (2016).

[18] T. F. Willems, C. H. Rycroft, M. Kazi, J. C. Meza, and M. Haranczyk, Microporous and Mesoporous Materials 149, 134 (2012).

[19] S. Chavan, J. G. Vitillo, D. Gianolio, O. Zavorotynska, B. Civalleri, S. Jakobsen, M. H. Nilsen, L. Valenzano, C. Lamberti, K. P. Lillerud, and S. Bordiga, Physical Chemistry Chemical Physics 14, 1614 (2012).

[20] K. Trepte, J. Schaber, S. Schwalbe, F. Drache, I. Senkovska, S. Kaskel, J. Kortus, E. Brunner, and G. Seifert, Physical Chemistry Chemical Physics 19, 10020 (2017). 
[21] K. Trepte, S. Schwalbe, J. Schaber, S. Krause, I. Senkovska, S. Kaskel, E. Brunner, J. Kortus, and G. Seifert, Physical Chemistry Chemical Physics 20, 25039 (2018).

[22] Q. Yang, V. Guillerm, F. Ragon, A. D. Wiersum, P. L. Llewellyn, C. Zhong, T. Devic, C. Serre, and G. Maurin, Chemical Communications 48, 9831 (2012).

[23] V. Bon, N. Klein, I. Senkovska, A. Heerwig, J. Getzschmann, D. Wallacher, I. Zizak, M. Brzhezinskaya, U. Mueller, and S. Kaskel, Physical Chemistry Chemical Physics 17, 17471 (2015).

[24] K. Trepte, S. Schwalbe, and G. Seifert, Physical Chemistry Chemical Physics 17, 17122 (2015).

[25] S. Schwalbe, K. Trepte, G. Seifert, and J. Kortus, Physical Chemistry Chemical Physics 18, 8075 (2016).

[26] M. Eddaoudi, J. Kim, N. Rosi, D. Vodak, J. Wachter, M. O'Keeffe, and O. M. Yaghi, Science 295, 469 (2002).

[27] N. L. Rosi, J. Eckert, M. Eddaoudi, D. T. Vodak, J. Kim, M. O'Keeffe, and O. M. Yaghi, Science 300, 1127 (2003).

[28] S. S.-Y. Chui, S. M.-F. Lo, J. P. H. Charmant, A. G. Orpen, and I. D. Williams, Science 283, 1148 (1999).

[29] C. H. Hendon and A. Walsh, Chemical Science 6, 3674 (2015).

[30] K. T. Butler, C. H. Hendon, and A. Walsh, Journal of the American Chemical Society 136, 2703 (2014).

[31] H. Furukawa, N. Ko, Y. B. Go, N. Aratani, S. B. Choi, E. Choi, A. Ö. Yazaydin, R. Q. Snurr, M. O'Keeffe,
J. Kim, and O. M. Yaghi, Science 329, 424 (2010).

[32] K. D. Gibson and H. A. Scheraga, Molecular Physics 62, 1247 (1987).

[33] R. D. Johnson, NIST Computational Chemistry Comparison and Benchmark Database (NIST Standard Reference Database Number 101, 19-04-2018).

[34] R. L. Martin, B. Smit, and M. Haranczyk, Journal of Chemical Information and Modeling 52, 308 (2012).

[35] M. Pinheiro, R. L. Martin, C. H. Rycroft, A. Jones, E. Iglesia, and M. Haranczyk, Journal of Molecular Graphics and Modelling 44, 208 (2013).

[36] M. Pinheiro, R. L. Martin, C. H. Rycroft, and M. Haranczyk, CrystEngComm 15, 7531 (2013).

[37] R. L. Martin and M. Haranczyk, Crystal Growth \& Design 14, 2431 (2014).

[38] H. Wu, T. Yildrim, and W. Zhou, The Journal of Physical Chemistry Letters 4, 925 (2013).

[39] J. A. Mason, M. Veenstra, and J. R. Long, Chemical Science 5, 32 (2014).

[40] M. G. Martin and J. I. Siepmann, The Journal of Physical Chemistry B 102, 2569 (1998).

[41] A. K. Rappe, C. J. Casewit, K. S. Colwell, W. A. Goddard, and W. M. Skiff, Journal of the American Chemical Society 114, 10024 (1992). 